\title{
Measurement of the total energy of an isolated system by an internal observer
}

\author{
S. Massar \\ Laboratoire d'Information Quantique and QUIC, C.P. 165/59, \\ Université Libre de Bruxelles, Av. F. D. Roosevelt, B-1050 Bruxelles, Belgium
}

\author{
S. Popescu \\ H. H. Wills Physics Laboratory, University of Bristol, Tyndall Avenue, Bristol BS8 1TL, U.K. and \\ Hewlett-Packard Laboratories, Stoke Gifford, Bristol BS12 6QZ, U.K.
}

(Dated: August 12, 2018)

\begin{abstract}
We consider the situation in which an observer internal to an isolated system wants to measure the total energy of the isolated system (this includes his own energy, that of the measuring device and clocks used, etc...). We show that he can do this in an arbitrarily short time, as measured by his own clock. This measurement is not subjected to a time-energy uncertainty relation. The properties of such measurements are discussed in detail with particular emphasis on the relation between the duration of the measurement as measured by internal clocks versus external clocks.
\end{abstract}

\section{INTRODUCTION}

The interpretation of quantum mechanics is based to a large extent on understanding the measurement process. This is because measurements are the interface between quantum systems and the observer. The present paper is devoted to the study of measurements in a very particular situation, namely we shall consider an observer inside an isolated system who attempts to measure the total energy of the isolated system. We shall be particularly interested in the time it takes the internal observer to measure the total energy. Our analysis sheds light on several troublesome aspects of quantum mechanics: the nature of observable quantities in quantum mechanics, the interpretation of energy measurements, the interpretation of isolated systems and the interpretation of time for such systems.

A central theme in our paper is the interpretation of the time-energy uncertainty relation in the context of energy measurements. Before delving into the specific subject of this article it is useful to recall what is known about this question. It was initially believed (in analogy with the situation for position and momentum analyzed by Heisenberg) that the precision $\Delta E$ with which the energy of a quantum system is measured and the time $T$ taken by the measurement are related by $T \Delta E \geq 1$ (where we take $\hbar=1$ ) [1]. However it was shown by Aharonov and Bohm 2] that this intuition is incorrect and that the energy of a quantum system can be measured in arbitrarily short time. (For a collection of reprints on quantum measurements, including the above two articles, see [3]). Recently it was realized that in order to carry out the fast measurement of Aharonov and Bohm the Hamiltonian must be known before hand. If the Hamiltonian is not known then the energy cannot be determined instantaneously, and in this case the precision $\Delta E$ with which the energy of a system is measured and the time taken for this measurement are constrained by $T \Delta E \geq 14$.

In the works described above the measurement is carried out by an external observer who couples the system to be measured to an external measuring device for a time $T$. Aharonov and Reznik [5] added a twist to this problem by asking whether an observer internal to an isolated quantum system can measure the total energy of the system itself. They showed that such internal measurements of the total energy are indeed possible. Aharonov and Reznik illustrate this by a simple example in which a self gravitating system, say a planet of radius $R$, ejects outwards a small mass $m$. By measuring the time $t$ it takes the mass to fall back onto the planet one can determine the mass of the planet and thus deduce its total energy $E$ through the correspondence between energy and mass $E=M c^{2}$. Aharonov and Reznik also present a more abstract mathematical model to illustrate this, see section $\amalg$ for a detailed presentation of this model.

One of the main points of the present work is to show that in discussing the time taken to measure the energy of an isolated system one should make a distinction between the time measured by the external observer (the external time $\left.t_{e x t}\right)$ and the time measured by the observer internal to the system (the internal time $t_{\text {int }}$ ). In particular when discussing the time energy uncertainty one should distinguish whether the duration of the measurement $T$ is measured in internal or external time. Note that having the time of the internal and external observer differ is completely compatible with the usual principles of physics, and in particular with the general relativity where different observers have in general completely different time variables. We discuss this point further below.

The works 2, 4, 5] complemented with the results obtained in the present article show that the relationship between the precision $\Delta E$ with which the energy of a system is measured and the time $T$ taken for this measurement is much richer than previously thought. Indeed there are many cases which can be considered according to whether the observer which carries out the measurement is internal or external to the system, according to whether the duration 


\begin{tabular}{|c|c|c|c|c|}
\hline $\begin{array}{c}\text { Observer who carries } \\
\text { out the measurement }\end{array}$ & $\begin{array}{c}\text { Time variable for which } \\
\text { the duration of the } \\
\text { measurement is minimized } \\
\text { (external or internal time })\end{array}$ & $\begin{array}{c}\text { Hamiltonian of the system } \\
\text { (known or unknown) }\end{array}$ & $\begin{array}{c}\text { Constraint on duration } \\
\text { of the measurement }\end{array}$ \\
\hline 1 & external & external & known & $T_{\text {ext }}$ arbitrarily small \\
\hline 2 & external & external & unknown & $T_{\text {ext }} \Delta E \geq 1$ \\
\hline 3 & external & internal & known & $?$ \\
\hline 5 & external & internal & unknown & $?$ \\
\hline 6 & internal & external & unknown & $?$ \\
\hline 7 & internal & external & known & $T_{\text {int }}$ arbitrarily small \\
\hline 8 & internal & internal & unknown & $?$ \\
\hline
\end{tabular}

TABLE I: Summary of relations between the precision $\Delta E$ with which the energy of a system is measured and the time $T$ taken for the measurement, according to whether the observer which carries out the measurement is internal or external to the system, according to whether the duration of the measurement is measured in internal or external time, and according to whether the Hamiltonian of the system is known or unknown.

of the measurement is measured in internal or external time, and according to whether the Hamiltonian of the system is known or unknown. Table \summarizes these different possibilities as well as what is known about them. Case 1 in the table is the situation analyzed in [2], case 2 is the situation analyzed in [4] and case 7 is the subject of the present article. We will also make some comments about case 5. Note that little or nothing is known about most of the cases in the table. Thus much more work is required to completely understand the status of the time-energy uncertainty relation in the context of energy measurements.

Let us now turn back to the case of the internal observer who wants to measure the total energy of an isolated system and who knows the Hamiltonian of the isolated system. In the particular examples they analyze Aharonov and Reznik find an intriguing effect, namely that the precision $\Delta E$ with which the total energy is measured and the time taken for the measurement are not independent. They find that they are related by $T \Delta E \geq 1$. On the basis of these examples they go on to argue that this is a fundamental constraint relating the precision with which the total energy of an isolated system is measured and the amount of time taken to carry out the measurement. A central result of the present article is to correct this statement.

Let us first note that if Aharonov and Reznik's claim was to be confirmed it would have important consequences for the interpretation of observable quantities in quantum mechanics because it would mean that there are quantities which are observable in principle, but are not observable instantaneously because they can only be measured in a finite time. This could give rise to some surprising (even paradoxical) situations $[\underline{8}]$ and is contrary to the situation concerning all other measurements of observables. For instance it is quite obvious that a position measurement can be done arbitrarily fast. And it was shown in [2] that any observable, and in particular the Hamiltonian observable, can be measured in arbitrary short time. (Note that the instantaneous measurements described in [2] require a measuring device with arbitrarily large energy external to the system to be measured. They therefore cannot be applied to measurements of the energy of an isolated system by an internal observer).

A clear understanding of measurements of total energy by an observer inside an isolated system therefore have direct bearing on the interpretation of quantum mechanics and in particular on what is meant by an observable quantity. In this paper we shall resolve the apparent paradox discovered by Aharonov and Reznik. To this end we must, as mentioned above, distinguish two notions of time for isolated systems. First there is the internal time $t_{\text {int }}$. This is the time as measured by a clock inside the isolated system. We shall show by an example that the precision $\Delta E$ with which the internal observer measures the total energy of the isolated system and the amount of internal time taken for the measurement $T_{\text {int }}$ are not mutually constrained. In particular $T_{\text {int }}$ can be much smaller than $1 / \Delta E$, see case 7 in the table. (Throughout this article we will denote by the capital letter $\mathrm{T}$ the duration of the measurement and by the small letter t the time variable.)

Second there is the external time $t_{\text {ext }}$. This is the time which would be measured by an observer outside the system. We do not know whether the precision $\Delta E$ with which the internal observer measures the total energy of the isolated system and the amount of external time taken for the measurement $T_{\text {ext }}$ are mutually constrained, although it is tempting to conjecture, see section $\square$ that in this case $T_{\text {ext }} \Delta E \geq 1$ holds. At present however we leave a ? in case 5 in the table.

The resolution of the apparent paradox uncovered in [5] is therefore that the internal observer can measure the total energy arbitrarily fast in his own proper time. Similarly the total energy of the isolated system can be measured 
by the external observer arbitrarily fast [2]. Thus the energy of an isolated system can be measured arbitrarily fast in the proper time of the observer, whether or not he is internal to the system or not.

As mentioned above having the internal and external times differ isn't in contradiction with basic physical principles. Indeed in general relativity the clocks of two systems have no reason to be synchronized, or to stay so during their evolution. We can thus imagine that the isolated system is a planet. For an observer at the surface of the planet and an observer at infinity, the times differ by the gravitational red-shift factor: $t_{\text {surface }}=\sqrt{g_{00}} t_{\infty}$. If $g_{00}$ is constant this is just a rescaling of time. But if $g_{00}$ changes during the energy measurement itself, than the amount of external time $T_{\infty}$ and the amount of internal time $T_{\text {surface }}$ taken for the measurement may differ in a non trivial way. Thus we can imagine that the measurement begins by a dynamical evolution during which $g_{00}$ decreases from its initial value to a very small value. This could for instance be due to a gravitational collapse of the planet which stops just above its Schwarzschild radius. Once $g_{00}$ is small the internal observable carries out the measurement of his total energy. Once the measurement is finished the planet expands and brings $g_{00}$ back to its initial value. This shows that the measurement can have very different durations for the internal and external observer. In section III we shall show that the duration for the internal observer can be arbitrarily short.

Furthermore $g_{00}$ is in fact an operator and can have uncertain value. This means that the relation between $t_{\text {surface }}$ and $t_{\infty}$, and hence the relation between internal and external time, becomes uncertain. This effect is inevitable and plays an essential role in understanding these measurements, as we discuss below.

In what follows we first review the mathematical model of Aharonov and Reznik and point out its limitations. We then introduce an alternative model in which the energy of an isolated system can be measured by an internal observer arbitrarily fast in his internal time. Finally we discuss its interpretation.

\section{THE MODEL OF AHARONOV AND REZNIK}

Aharonov and Reznik consider an isolated system containing a clock. The system is described (in the terminology of [5]) by the Hamiltonian $H_{c}+H_{b o x}$ where $H_{c}=-i \partial_{x}=p_{x}$ is the Hamiltonian of the internal clock variable $x$ $\left(\left[x, p_{x}\right]=i\right)$ and $H_{b o x}$ is the Hamiltonian of the rest of the isolated system. For simplicity we take $H_{b o x}$ to be independent of $x$, but the analysis can be easily generalized to the case where it depends on $x$.

In addition the isolated system contains a measurement variable $q$ with conjugate momentum $p$. The outcome of the measurement will be recorded in the value of $p$. The measurement only takes place for $x_{i} \leq x \leq x_{f}$. Thus for $x<x_{i}$ or $x>x_{f}$ the measuring device is uncoupled to the system and the total Hamiltonian is $H_{c}+H_{b o x}$ (we take for simplicity the free Hamiltonian of the measuring device to be zero) whereas between $x_{i} \leq x \leq x_{f}$ the measuring

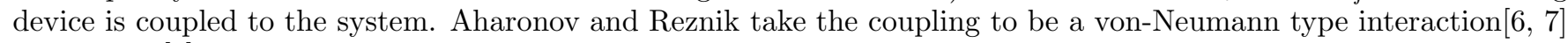
of the form $[9]$ :

$$
H=H_{c}+H_{b o x}+\left(\frac{1}{2} g(x) H_{c}+\frac{1}{2} H_{c} g(x)+g(x) H_{b o x}\right) q .
$$

Here $g(x)$ is the coupling function which is non zero only when $x_{i} \leq x \leq x_{f}$. We take $g(x)$ to increase rapidly from zero to $g$ when the measurement starts, then to stay constant during the measurement, and to decrease rapidly to zero when the measurement ends, see fig. 1 . The duration of the measurement, as measured by the $x$ variable, will be denoted $L=x_{f}-x_{i}$.

Let us suppose the system is in an eigenstate of the total energy

$$
H|\Psi\rangle=E_{0}|\Psi\rangle
$$

that the box is in an eigenstate of $H_{b o x}$

$$
H_{b o x}\left|u_{E_{b o x}}\right\rangle=E_{b o x}\left|u_{E_{b o x}}\right\rangle,
$$

and that the measuring device is in an eigenstate of the measurement variable $q$. Then the state of the isolated system can be written as

$$
|\Psi\rangle=\psi\left(x, E_{0}, E_{b o x}, q\right)|q\rangle\left|u_{E_{b o x}}\right\rangle .
$$

Substituting into eq. (11) one obtains the exact solution:

$$
|\Psi\rangle=\frac{1}{\sqrt{1+g(x) q}} e^{-i E_{b o x} x} e^{i E_{0} \int^{x} \frac{d x^{\prime}}{1+g\left(x^{\prime}\right) q}}|q\rangle\left|u_{E_{b o x}}\right\rangle .
$$


The internal time is

$$
t_{\text {int }}=x
$$

since this is the variable that multiplies $E_{\text {box }}$. Indeed if one realizes a superposition of different states with different $E_{\text {box }}$, then the internal state of the box will evolve in time $x=t_{\text {int }}$.

On the other hand the external time, expressed as function of the variables of the isolate system, is

$$
t_{\text {ext }}(x, q)=\int^{x} \frac{d x^{\prime}}{1+g\left(x^{\prime}\right) q}
$$

since this is the variable that multiplies the total energy $E_{0}$. Indeed if one realizes a superposition of states with different values of $E_{0}$, then one will find that $t_{\text {ext }}(x, q) \simeq t$ (where the wavefunction is solution of $i \partial_{t} \Psi=H \Psi$, ie. $t$ is the absolute external time which can be measured to arbitrary high precision by an external observer with sufficient energy).

In order to analyze the solution eq. (5) Aharonov and Reznik suppose that $g(x) q<<1$ and then expand the phase in eq. (5) to first order in $g(x) q$. Note that when $g(x) q<<1$, the external time and the internal time coincide $t_{\text {ext }}=t_{\text {int }}$ and the two need no longer be distinguished. As we shall see in section III these times need not necessarily coincide.

One of the points of the following discussion is to show that the first order expansion of Aharonov and Reznik is not always legitimate. To illustrate this we shall keep the second order terms in the phase. Thus we approximate eq. (5) as

$$
|\Psi\rangle=\frac{1}{\sqrt{1+g(x) q}} e^{-i E_{b o x} x} e^{i E_{0} \int^{x} d x^{\prime}\left(1-g\left(x^{\prime}\right) q+g^{2}\left(x^{\prime}\right) q^{2}\right)}|q\rangle\left|u_{E_{b o x}}\right\rangle .
$$

After the measurement, ie. when $x>x_{f}$, and using the fact that $\int_{x_{i}}^{x_{f}} d x^{\prime} g^{n}\left(x^{\prime}\right) \simeq L g^{n}$ (see the form of $g(x)$ in fig. 1) we have

$$
|\Psi\rangle=e^{-i E_{b o x} x} e^{i E_{0} x} e^{-i E_{0} L g q+i E_{0} L g^{2} q^{2}}|q\rangle\left|u_{E_{b o x}}\right\rangle, x>x_{f}
$$

Let us now suppose that initially the measuring device is in a Gaussian state $N e^{-q^{2} / 2 \sigma^{2}}$ centered on $q=0$ with width $\Delta q=\sigma$ ( $N$ is the normalization constant). Then at late times the state becomes

$$
\begin{aligned}
|\Psi\rangle & =\int d q N e^{-q^{2} / 2 \sigma^{2}} e^{i\left(E_{0}-E_{b o x}\right) x} e^{-i E_{0} L g q+i E_{0} L g^{2} q^{2}}|q\rangle\left|u_{E_{b o x}}\right\rangle \\
& =N^{\prime} e^{i\left(E_{0}-E_{b o x}\right) x} \int d p \exp \left[-\frac{\left(p-L g E_{0}\right)^{2}}{2} \frac{\sigma^{2}}{1+4 L^{2} g^{4} E_{0}^{2} \sigma^{4}}\left(1+i 2 L g^{2} E_{0} \sigma^{2}\right)\right]|p\rangle\left|u_{E_{b o x}}\right\rangle
\end{aligned}
$$

where we have rewritten the state in momentum representation.

Thus we see that the mean value of $p$ has been displaced by

$$
p \rightarrow p+L g E_{0} \text {. }
$$

This means that a measurement of the total energy of the isolated system has indeed taken place and that its value is registered in the pointer $p$.

The spread in the value of $p$ is given by eq. (8):

$$
\Delta p=\frac{1}{\sigma} \sqrt{1+4 L^{2} g^{4} E_{0}^{2} \sigma^{4}} .
$$

The precision $\Delta E_{0}$ with which the energy is measure is thus

$$
\Delta E_{0}=\frac{1}{L g \sigma} \sqrt{1+4 L^{2} g^{4} E_{0}^{2} \sigma^{4}} .
$$

In discussing the precision of the measurement we must thus distinguish two cases according to the values of $E_{0}$.

1. $\left|E_{0}\right|<1 / 2 L g^{2} \sigma^{2}$. In this case $\Delta E_{0} \simeq 1 / L g \sigma$. We recall that $g \sigma<<1$ (this is the condition for the expansion in $g(x) q$ in the phase to be valid) and that $L=T$ is the duration of the measurement (we do not distinguish between internal and external time since when $g \sigma<<1$ they coincide) to obtain

$$
T \Delta E_{0}>>1 \text {. }
$$

Note that one can come close to saturating this relation when $g \sigma$ is not very small compared to 1 . 
2. $\left|E_{0}\right|>1 / 2 L g^{2} \sigma^{2}$. In this case it is the second term under the square root in eq. (10) which dominates and one cannot even come close to saturating eq. (11). In fact when $\left|E_{0}\right|$ increases one is further and further from saturating eq. (11).

Several remarks are now in order. First of all the parameters $g, \sigma, L$ can be chosen by the internal observer. But once they are chosen they are fixed. This means that there will be only a finite band of energy for which eq. (11) can be approximately saturated. As the energies get further and further from this band one is further and further from saturating this inequality. This means that eq. (11) does not have a fundamental character in the context of the model of [5].

Second the initial state chosen above $e^{-q^{2} / 2 \sigma^{2}}$ implies that the resolution of the measurement is maximal near $E_{0}=0$. By putting a phase on the initial state one can change the energy at which the which the resolution comes close to saturating eq. (11). But it will always be optimal in an energy band only.

Third in the above calculation we took a Gaussian initial state and expanded the phase to second order so as to be able to do an exact calculation. It is not difficult to persuade oneself -for instance by using an analogy with the effect of dispersion on propagating waves- that the general conclusion, namely that the time energy uncertainty relation eq. (11) can only be saturated in an energy band, continues to hold for arbitrary states and when the phase is expanded to higher orders in $g q$.

In summary there are two problems with the conclusions of [5] concerning the time energy uncertainty. First of all it is only a model, and one should not draw universal conclusions about the validity of the time-energy uncertainty from a single model. Secondly in this model the time-energy uncertainty, although it is obeyed, does not appear as fundamental since for most values of the energy is cannot be saturated.

\section{A MODEL WHICH ALLOWS INTERNAL OBSERVERS TO MEASURE THE TOTAL ENERGY ARBITRARILY FAST}

We now introduce an alternative model that allows an internal observer to carry out measurements of the total energy arbitrarily fast in his own internal time. The Hamiltonian of the box, the clock and the measuring device is:

$$
H=\frac{H_{b o x}}{1+q g(x)}+\frac{1}{2}\left(\frac{1}{1+q g(x)} H_{c}+H_{c} \frac{1}{1+q g(x)}\right)
$$

where the notation is the same as in section [II eq. (1).

Note that this Hamiltonian can be thought of as describing the isolated system undergoing gravitational collapse as discussed in the introduction. Indeed the Hamiltonian of such a collapsing planet would be $H=\sqrt{g_{00}} H_{p l a n e t}$. Our model thus corresponds to taking the metric to be $\sqrt{g_{00}}=1 /(1+q g(x))$. Note that this metric is an operator (since $q$ and $x$ are operators). This will play a crucial role in what follows.

The exact solution of the Schrödinger equation $H|\Psi\rangle=E_{0}|\Psi\rangle$ is

$$
|\Psi\rangle=\sqrt{1+g(x) q} e^{-i E_{\text {box }} x} e^{i E_{0} \int^{x} d x^{\prime}\left(1+g\left(x^{\prime}\right) q\right)}|q\rangle\left|u_{E_{\text {box }}}\right\rangle
$$

where the notation is once more as in section [II eq. (5).

In the present case the internal time is

$$
t_{\text {int }}=x
$$

and the external time, expressed as function of the variables of the isolate system, is

$$
t_{\text {ext }}=\int^{x} d x^{\prime}\left(1+g\left(x^{\prime}\right) q\right)
$$

since these are the functions that multiply $E_{b o x}$ and $E_{0}$ respectively in the phase of $\Psi$. Note that before and after the measurement $g=0$ and the internal and external time coincide. But we will allow them to be very different during the measurement.

After the measurement, when $x>x_{f}$, the solution takes the form (where we use the form of $g(x)$ given in fig. 1):

$$
|\Psi\rangle=e^{-i E_{\text {box } x} x} e^{i E_{0} x} e^{i E_{0} L g q}|q\rangle\left|u_{E_{b o x}}\right\rangle, x>x_{f}
$$

Thus after the measurement the momentum $p$ of the measuring device has been displaced by

$$
p \rightarrow p-\operatorname{Lg} E_{0}
$$


Note that no approximations have been made in obtaining this result, contrary to the the way eq. (9) is obtained in the model of Aharonov and Reznik. If the momentum of the measuring device has spread $\Delta p$, then eq. (17) constitutes a measurement of total energy with precision

$$
\Delta E_{0}=\frac{\Delta p}{L g} .
$$

In order to interpret eq. (18) we must specify the initial state of the measuring device. We will take the initial state to only have values $q>0$. This ensures (since $g>0$ ) that the Hamiltonian eq. (12) never becomes infinite and that the model is well defined. We will thus suppose that the initial state is centered on $\bar{q}$ with width $\Delta q$ such that $\bar{q}>>\Delta q$. Note that these constraints are compatible with taking the initial state to be almost Gaussian and thus to almost saturate the inequality $\Delta q \Delta p \geq 1$. The constraint on the precision of the measurement can be rewritten as

$$
\Delta E_{0} \geq 1 /(L g \Delta q)
$$

and this inequality can be almost saturated in the case of the quasi Gaussian states just mentioned.

The amount of internal time it takes to carry out the measurement is $T_{i n t}=L$. We can choose $L$ arbitrarily small. We can also choose $g$ such that $L g \Delta q$ is arbitrarily large. Thus the internal observer can measure the total energy of the system to arbitrarily high precision in arbitrarily small internal time. This is our main result.

\section{THE RELATION BETWEEN INTERNAL AND EXTERNAL TIME.}

Now we come to an interesting point, namely how the external time variable $t_{e x t}$ is related to the internal time variable $t_{\text {int }}$ and how the duration of the measurement, as measured by the two observers, are related. Let us imagine that before the measurement starts both clocks are synchronized and that the measurement starts at $t=0$ as indicated by both clocks. As soon as the measurement is finished, the internal observer sends a signal to the external observer. What will be the time $T_{\text {ext }}$ indicated by the external clock?

To answer this question we use eqs. (14) and (15) to find

$$
T_{\text {ext }}=T_{i n t}+T_{i n t} g q .
$$

$T_{\text {int }}$ is well defined. However $q$ is an operator and has uncertainty. Therefore $T_{\text {ext }}$ is uncertain. This raises two questions: what is its average value $\overline{T_{\text {ext }}}$ and what is its spread $\Delta T_{\text {ext }}$ ?

The uncertainty is given by $\Delta T_{e x t}=L g \Delta q$. The constraint eq. (19) can thus be interpreted as a relation between the precision of the measurement and the uncertainty in the duration of the measurement as measured in external time:

$$
\Delta E_{0} \Delta T_{\text {ext }} \geq 1 .
$$

We will argue below that this constraint is universal and must apply to all measurements of total energy by an internal observer.

The average duration of the measurement is $\overline{T_{e x t}}=T_{\text {int }}(1+g \bar{q})$. Since in our model $\Delta q<<\bar{q}$ the average duration of the measurement, as measured in external time, is constrained by

$$
\Delta E_{0} T_{e x t} \geq 1 .
$$

However as discussed below we are not sure whether this constraint is universal.

We now address the universality of eqs. (21) and (22). To this end let us view the total system consisting of the internal and the external system as a whole. Let us suppose that the total system is in an energy eigenstate. Let us also suppose that initially the internal and external times are correlated (this is possible since $t_{\text {ext }}-t_{\text {int }}$ commutes with the total energy $\left.E_{\text {total }}\right)$. Then the internal measurement of the internal energy can also be viewed as saying that the internal observer is measuring the energy of the external system.

But we know that when an external observer measures the energy of a system the internal clock gets randomized (since the energy of the system and the clock variable are conjugate operators in the usual sense). Now in the previous example the internal observer can be considered "external" to the original external system which now becomes the "internal" system. Hence the original internal observer's measurement must randomize the original external time. Thus eq. 21) must always hold.

Does this imply that eq. (22) is also universal? At first sight one would think so. But so far we have been unable to find a proof. (For instance it seems logically possible -although unlikely- that one could devise a model in which the measurement almost always takes a very short external time, and very rarely takes a very long external time, in such a way that the average duration of the measurement is arbitrarily short, but the spread in the durations obeys eq. (21). Such a model would not satisfy eq. (22)). We thus leave the universal validity of eq. (22) as a conjecture. 


\section{CONCLUSION}

We have shown that it is possible for an internal observer to measure the total energy of an isolated system in arbitrary short internal time. This was done in a particular model. An interesting question is whether one can make the model more realistic, for instance by basing it on the model of gravitational collapse discussed in the introduction. In this respect one problem pointed out to us by Y. Aharonov [8] is that in our model the Hamiltonian is not bounded from below. We are not sure to what extent taking a positive Hamiltonian will modify our conclusions. Preliminary investigations suggest that our main conclusions will remain unchanged.

Acknowledgment. We thank Yakir Aharonov for enlightening discussions. We acknowledge financial support from the European Union through project RESQ IST-2001-37559, from EPSRC U.K. through QIPIRC, from the Action de Recherche Concertée de la Communauté Française de Belgique, from the IUAP program of the Belgian Federal Government under grant V-18.

[1] L. Landau and Peierls, Z. Physics 69 (1931) 56

[2] Y. Aharonov and D. Bohm, Phys. Rev. 122 (1961) 1649

[3] J. A. Wheeler and W. H. Zurek (Eds), Quantum Theory and Measurement, Princeton, N.J.: Princeton University Press, 1983

[4] Y. Aharonov, S. Massar, S. Popescu, Phys. Rev. A 66, 052107 (2002)

[5] Y. Aharonov and B. Reznik, Phys. Rev. Lett. 84 (2000) 1368

[6] J. von Neumann, Mathematical Foundations of Quantum Mechanics, Princeton University Press, reprint edition 1996

[7] D. Bohm, Quantum Theory, Dover Publications 1989

[8] Y. Aharonov, private communication

[9] Recall that for measuring a variable $A$ the standard von Neumann interaction Hamiltonian is $g(t) A q$ where $t$ is the time variable, ie. a classical parameter. In our case however the role of time is played by the clock pointer $x$, so that $g(t) \rightarrow g(x)$. Furthermore in our case $A=H_{c}+H_{b o x}$. Since $g(x)$ does not commute with $H_{c}$ the interaction Hamiltonian has to be symmetrised: $g(x) H_{c} \rightarrow\left(g(x) H_{c}+H_{c} g(x)\right) / 2$.

Figure 1: caption The function $g(x)$ that controls when the measuring device is coupled to the total energy. 
<smiles>[CH]1CC[Al]1</smiles> 